\title{
Boltzmann equations and ab initio calculations: comparative study of cubic and wurtzite CdSe
}

\author{
A. Abbassi", Z. Zarhri, Ch. Azahaf, H. Ez-Zahraouy and A. Benyoussef
}

${ }^{*}$ Correspondence: Abbassi. abder@gmail.com

Laboratory of Magnetism and High Energy Physics (URAC 12), Faculty

of Sciences, Mohammed V University, B.P. 1014, Rabat, Morocco

\begin{abstract}
The electronic and optical properties of CdSe in two phases, cubic and wurtzite, have been studied by first principal calculations using the density functional theory. The optical parameters such as transmittance, optical absorption, refractive index and extinction coefficient have been investigated. We have calculated also the band structure, and total/partial density of state using the full potential-linearized augmented plane wave method with the local density approximation, generalized gradient approximation and the modified Becke-Johnson functional (mBJ), implemented in the Wien2k package. With the $\mathrm{mBJ}$ approximation the gap found for cubic and wurtzite structure is direct and is equal to 1.85 and $1.7 \mathrm{eV}$ respectively, what corresponds to the experiment results. The optical absorption is significant in the ultraviolet field while it becomes low beyond $600 \mathrm{~nm}$ in the visible light for $\mathrm{CdSe}$ in different structures. From $\lambda=400 \mathrm{~nm}$ the transmittance is stable and reaches $80 \%$. With Boltztrap package, we have investigated also that with increasing temperature, the electrical conductivity increases. During the calculation, the cubic structure has presented an isotropy. While for wurtzite CdSe, the propagation of waves into system is different in xx and zz directions. These results can be exploited in several applications of CdSe in optoelectronic devices.
\end{abstract}

Keywords: DFT, Optical properties, CdSe, Wien2K, Boltzmann, Transmittance, Optical absorption, Band structure, Optoelectronic

\section{Background}

The semiconductor CdSe is type II-VI, consisting of an element of column II in the periodic table and an element from column VI. The cadmium atom is type II, it has two electrons in the valence band in orbital s, cd ( $4 \mathrm{~d} 105 \mathrm{~s} 2)$. The Selenium atom is material of type VI, it has six valence electrons in the s and p orbitals: 3d104s24p4. The valence band of the massive CdSe crystal consists essentially of the selenium p orbitals and the conduction band, of the cadmium orbital s. The massive CdSe material exists in two structures, cubic and wurtzite. The unit cell of $\mathrm{Cd}$ and $\mathrm{Se}$ is a hexagonal packing. The other form is the cubic structure, where the unit cell is cubic face-centered.

This type of semiconductors is considered an excellent candidate in optical devices technology, optical memories with high-density, transparent conductors, laser devices (Klude et al. 2002), photodetectors (Matsumura et al. 2002; Uthana and Reddy 1981)

\section{望 Springer}

(c) 2015 Abbassi et al. This article is distributed under the terms of the Creative Commons Attribution 4.0 International License (http://creativecommons.org/licenses/by/4.0/), which permits unrestricted use, distribution, and reproduction in any medium, provided you give appropriate credit to the original author(s) and the source, provide a link to the Creative Commons license, and indicate if changes were made. 
photoelectric sensors (Fuentes-Hernandez et al. 2004), solar cells (Merad et al. 2005; Merad et al. 2006; Karl and Jr 1982; Murray et al. 1993). It can be also used as a biological labeling (Jr et al. 1998), and in spintronic devices (Beaulac et al. 2008). The CdSe is transparent in infrared radiation, it is used sometimes in the fabrication of photoresistors and in thin layers for instruments using infrared light. This material is also highly luminescent.

CdSe crystallizes in two structures.

A hexagonal one, with space group P63 mc and the lattice parameters $\mathrm{a}=\mathrm{b}=4.299 \mathrm{~A}^{\circ}$ and $\mathrm{c}=7.010 \mathrm{~A}^{\circ}$. A cubic one, with space group F-43m and $\mathrm{a}=\mathrm{b}=\mathrm{c} 6.05 \mathrm{~A}^{\circ}$.

Several studies and research were treated CdSe using WIEN2k, treating its electronic properties, gap band (Akinci et al. 2009). The aim of this work is to investigate the electronic (DOS, PDOS, band structure), optical (absorption, transmittance, extinction factor, refractive index) and electrical (electrical conductivity) properties in both, CdSe Cubic and wurtzite phases. We will show that we can use CdSe in several applications of opto-electronic and photovoltaic.

This study is also a comparison between the two phases, we will demonstrate that our results obtained with this approximation mbj are in agreement with the experimental data.

\section{Calculation method}

The present calculation is based on ab initio electronic structure. The first principles DFT calculations have been made with the full potential linearized augmented plane wave (FP LAPW) method using the WIEN2k program (Blaha et al. 2011). We also have to mention that our calculations have been performed using different approximations GGA (Perdew et al. 1996), LDA (Slater 1951) and modified Becke- Johnson functional (Blaha and Schwarz 2006).

In order to define the calculation of wave function, we used an energy cutoff of -8.0 Ryd for the LAPW. In this calculation we work with the $500 \mathrm{~K}$-point and the self-consistent was found stable and converge at $10^{-6}$ Ryd.

The dielectric function is given by the following expression (Abbassi et al. 2015):

$$
\varepsilon(\omega)=\varepsilon_{1}(\omega)+i \varepsilon_{2}(\omega)=N^{2}
$$

with

$$
N(\omega)=n(\omega)+i k(\omega)
$$

The dielectric function comprises two terms: real and imaginary, these two terms describe the variation of optical parameters, the imaginary part of this function is related to the optical conductivity by the following equation:

$$
\varepsilon_{2}=\frac{\omega \sigma}{\varepsilon_{0}\left[\tau_{p}^{2}\left(\omega_{0}^{2}-\omega^{2}\right)^{2}+\omega^{2}\right]}
$$

The real part $\varepsilon_{1}(\omega)$ allows also the calculation of other optical parameters, namely, the refractive index and extinction coefficient or attenuation K-factor, which describes the energy loss of a radiation passing through the materials, this relation is given as follows: 


$$
\varepsilon_{1}=n^{2}-k^{2}
$$

It is also known that the absorption coefficient is related to the extinction coefficient what can be described by the following relation:

$$
\alpha=2 \pi k / \lambda
$$

To investigate the transport properties of $\mathrm{CdSe}$, we exploit the structure band data calculated with $\mathrm{Ab}$ initio method and fitted into Boltzmann package, using the Boltzmann theory and the approach of rigid band (Madsen and Singh 2006; Ziman 2001). We can learn from these approaches that the conductivity that depends on transport distribution can be given by:

$$
\sigma_{\alpha \beta}(\varepsilon)=\frac{1}{N} \sum_{i, k} \sigma_{\alpha \beta}(i, k) \frac{\delta\left(\varepsilon-\varepsilon_{i, k}\right)}{\delta(\varepsilon)}
$$

We have noticed that $\alpha$ and $\beta$ are the tensor indices, $\varepsilon_{i, k}$ is the band structure. $\mathrm{N}$ denotes the number of k-points that are sampled in the BZ.

\section{Electronic properties}

\section{Band structure, total and partial DOS}

Figure 1 shows the band structures of CdSe in different Cubic and hexagonal structures. This study was realized with different GGA, LDA and mBJ approximation. It is shown that the gap is direct in $\Gamma$ point for the cubic and hexagonal structures. These different approximations give different gap values. Therefore the gap value with the $\mathrm{mBJ}$ (modified beak-Johnson) is close to the value of the experimental data (Kale and Lokhande 2005). The potential of semi local exchange, which retrieves the local density approximation (LDA) and GGA to a constant electron density, leads to the calculations that are more accurate. Table 1 shows the different values of the gap obtained.

We have noted that both structures present DOS with presence of different regions of bands and energy levels. In the case of the cubic structure we observe that a very intense
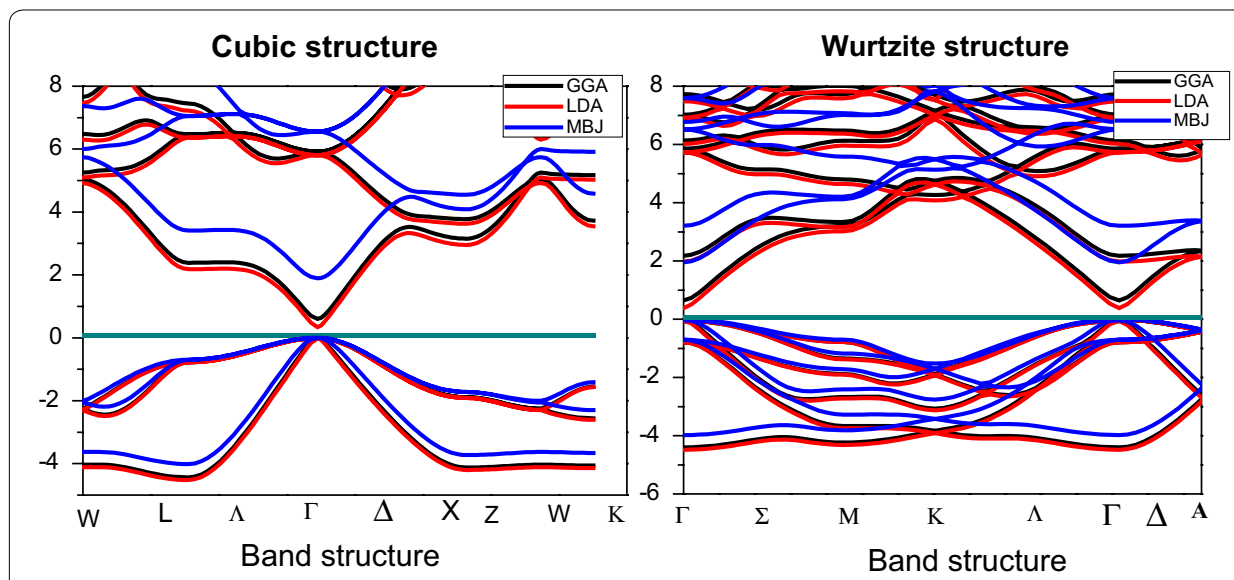

Fig. 1 Band structure of cubic and wurtzite CdSe 
Table 1 The different values of the gap energy

\begin{tabular}{lllll}
\hline & LDA $(\mathbf{e V})$ & GGA $(\mathbf{e V})$ & $\mathbf{m B J}(\mathbf{e V})$ & Experiment \\
\hline Gap-cubic phase & 0.412 & 0.72 & 1.85 & 2.3 Kale and Lokhande (2005) \\
Gap-Wurtzite phase & 0.367 & 0.634 & 1.7 & 1.7 Kale and Lokhande (2005) \\
\hline
\end{tabular}

region appears in the conduction band and especially from $4.5 \mathrm{eV}$. The hexagonal structure as shown in Fig. 2, presents a region with a very intense energy levels in valence and the conduction band. We will make a detailed calculation in the total and partial density of states in order to understand this electronic structure.

Figure 3 shows the total and partial density of state of CdSe in different structures. For the cubic structure it has several regions where energy level appears. In the valence band we can note that an intense band appear from -3.89 to $0 \mathrm{eV}$ mainly due to the orbital $\mathrm{p}$ of Se and another range from -8.04 to $-7.09 \mathrm{eV}$ mainly due to the orbital $\mathrm{d}$ of $\mathrm{Cd}$. The conduction band also has an intense range beyond $1.85 \mathrm{eV}$ mainly due to the orbital $\mathrm{d}$ of Se and low occurrence of $\mathrm{p}$ and $\mathrm{d}$ of Se.

In the hexagonal structure, the valence band is formed of an intense region from -3.89 to $0 \mathrm{eV}$ essentially due to the orbital $\mathrm{p}$ of $\mathrm{Se}$ and a region from -8.04 to $-7.04 \mathrm{eV}$ which is mainly due to the orbital $\mathrm{d}$ of $\mathrm{Cd}$. Beyond $1.7 \mathrm{eV}$, the conduction band is formed with a low occurrence of orbital d of $\mathrm{Cd}$ and $\mathrm{p}, \mathrm{d}$ of Se.

It is also noted that the difference between the gap energy of wurtzite and cubic structure is $\Delta \mathrm{Eg}=0.15 \mathrm{eV}$.

\section{Optical and transport properties}

Figure 4 shows the variation of absorption as a function of a wavelength for CdSe in different structures. This study shows that CdSe in the cubic structure is isotropic. The propagation of radiation $\mathrm{h} v$ in $\mathrm{xx}$ and $\mathrm{zz}$ directions is the same in the system. It has a significant absorption in the ultraviolet range and becomes very low from $600 \mathrm{~nm}$. In

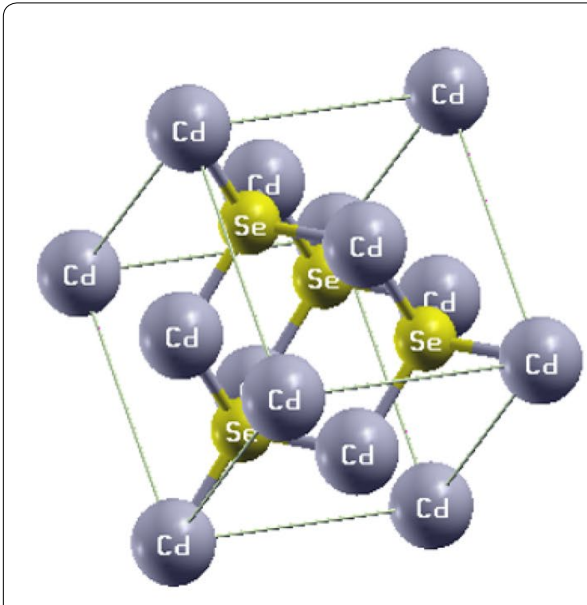

Cubic structure

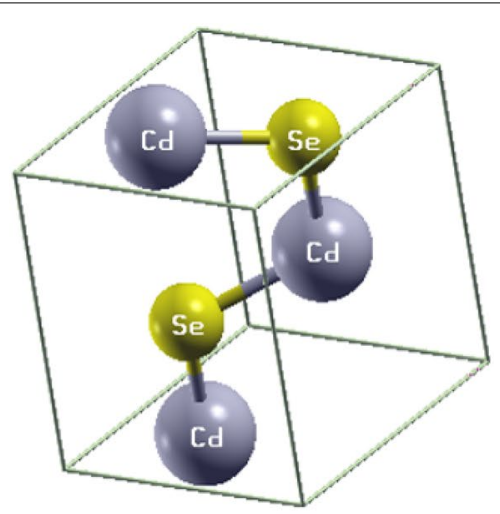

Wurtzite structure

Fig. 2 CdSe structures, cubic and wurtzite 


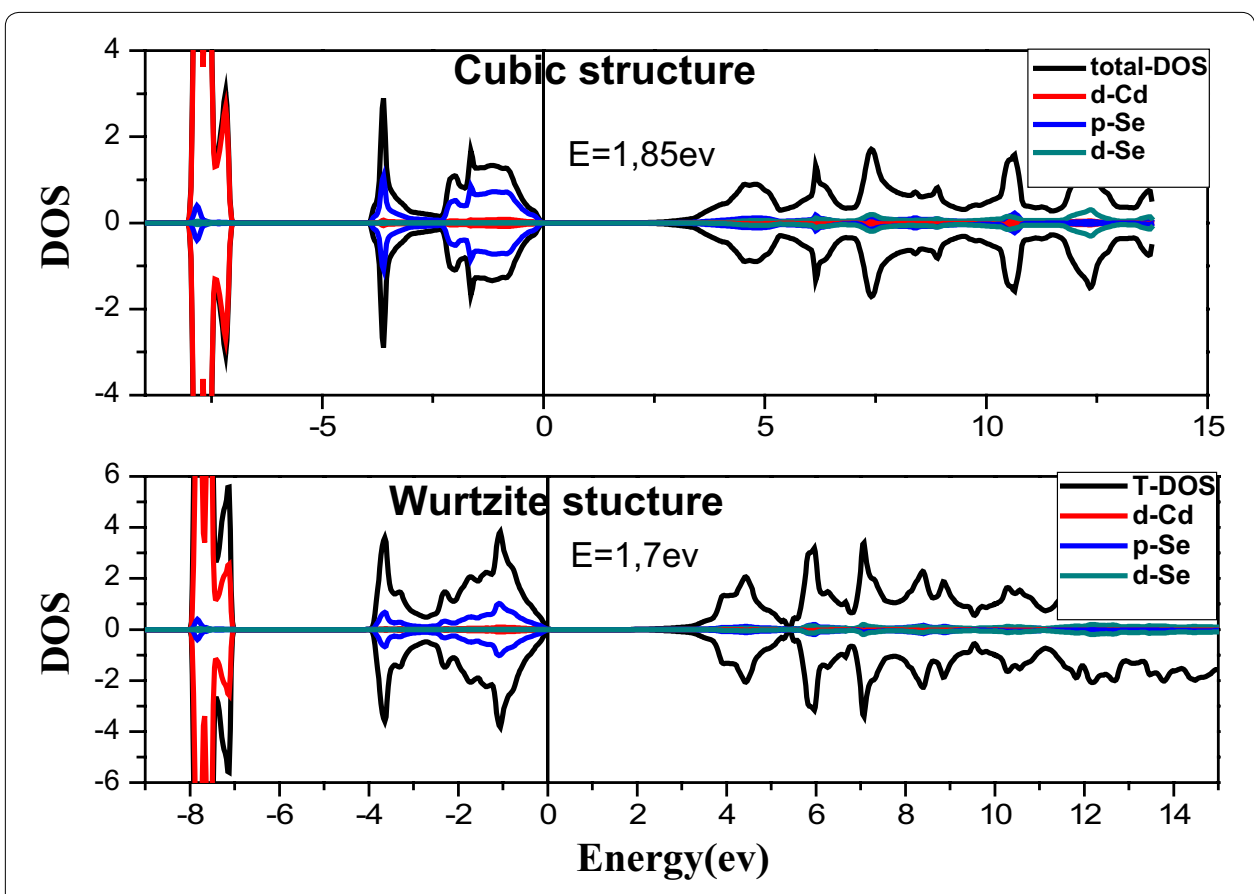

Fig. 3 Total and partial density of state
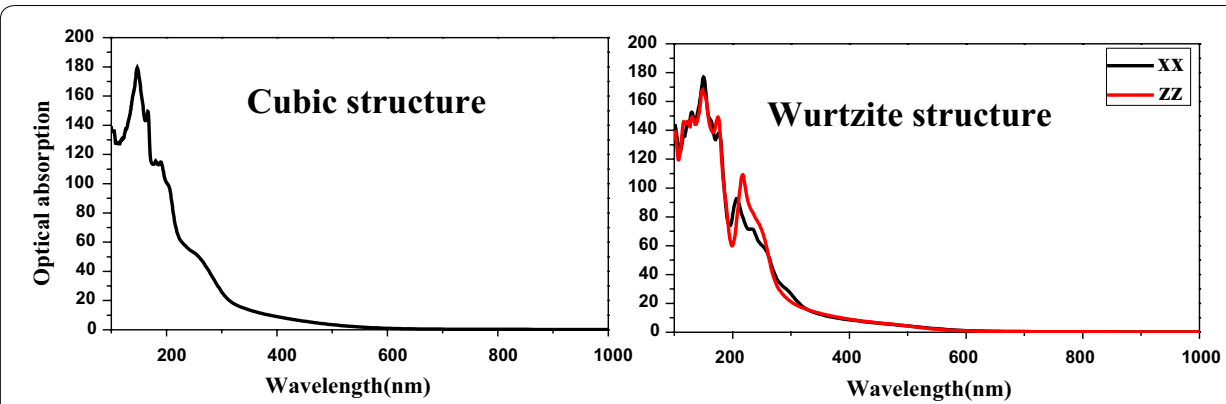

Fig. 4 Optical absorption of CdSe in different structures

the range of 380-600 $\mathrm{nm}$ in the visible light, CdSe has a low absorption and substantially lower than the ultraviolet.

CdSe in wurtzite structure is not completely isotropic, the propagation of radiation in the direction $x x$ and $z z$ is different, especially in the range of $200-300 \mathrm{~nm}$. The variation of the absorption in the region of ultraviolet and visible light of Wurtzite does not present a large difference with the cubic structure.

According to the Fig. 5, the transmittance of CdSe is stable and can reach $80 \%$ from $\lambda=400 \mathrm{~nm}$ for the cubic and hexagonal structures. The hexagonal system is not isotropic, it does not present the same variation of transmittance, particularly in the ultraviolet field.

In the ultraviolet field, the transmittance is not stable and shows the lowest value for the cubic structure at $\lambda=144 \mathrm{~nm}$ where the reached value amounts $40 \%$. CdSe in its 


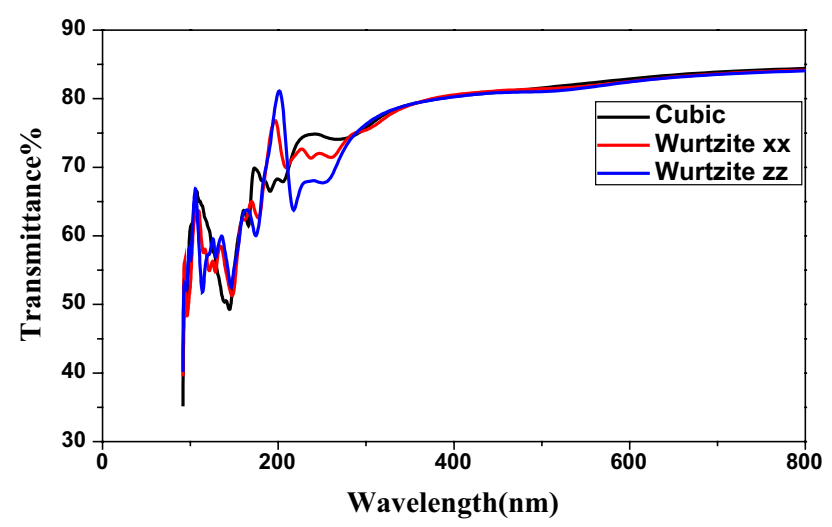

Fig. 5 Transmittance of CdSe in different structures

structure behaves as transparent in the visible light and absorbent in ultraviolet. These optical properties can be exploited in various optical applications.

The refractive index is also one of the optical properties of the systems, it describes the behavior of radiation propagating in materials. We have noted that in the absorbent systems, the refractive index consists of two expressions, real and imaginary parts as shown in Eq. (2).

It is also noted that the imaginary part of the refractive index shows the attenuation $\mathrm{K}$ of the wave, which is essentially designed to investigate the energy loss of the radiation. It is related to the absorption coefficient by the Eq. (5).

Figure 6 shows the variation of the refractive index $\mathrm{n}$ and the extinction coefficient $\mathrm{K}$ as a function of energy. The curves show that for the energies lower than $4 \mathrm{eV}, \mathrm{CdSe}$ is transparent, the highest values reached of the refractive index are: 2.78 for the cubic structure what corresponds to $4.2 \mathrm{eV}$ energy, and for the hexagonal structure the $\mathrm{n}=3.06$ what corresponds to the $4.68 \mathrm{eV}$ energy. The refractive index decreases in both structures with increasing of energy which is in agreement with these experiment results (Ninomiya and Adachi 1995). Regarding to the extinction coefficient, it increases to a value of 2.09 what corresponds to $\mathrm{E}=8.36 \mathrm{eV}$ for cubic structure and to $\mathrm{K}=2.11$ what correspond to $8.12 \mathrm{eV}$ in wurtzite structure (Fig. 7).

Using the Boltzmann equation mentioned above, we have calculated the variation of the electrical conductivity as a function of temperature. This calculation is very important to describe the electrical behavior of CdSe,

It has been observed that CdSe in cubic and wurtzite structure has an increasing electrical conductivity while the temperature increases, which means that the number of free electrons increases. Our results are in agreement with the experimental results (Suresh and Arunseshan 2014). This conductivity is mainly due to the presence of selenium vacancies as is reported by several researchers. Therefore, we can conclude that CdSe can be exploited as a semiconductor in photovoltaic applications, the conductivity and transmittance which reaches $80 \%$ are significant parameters that allow us to consider this material as a candidate in solar cell applications. 

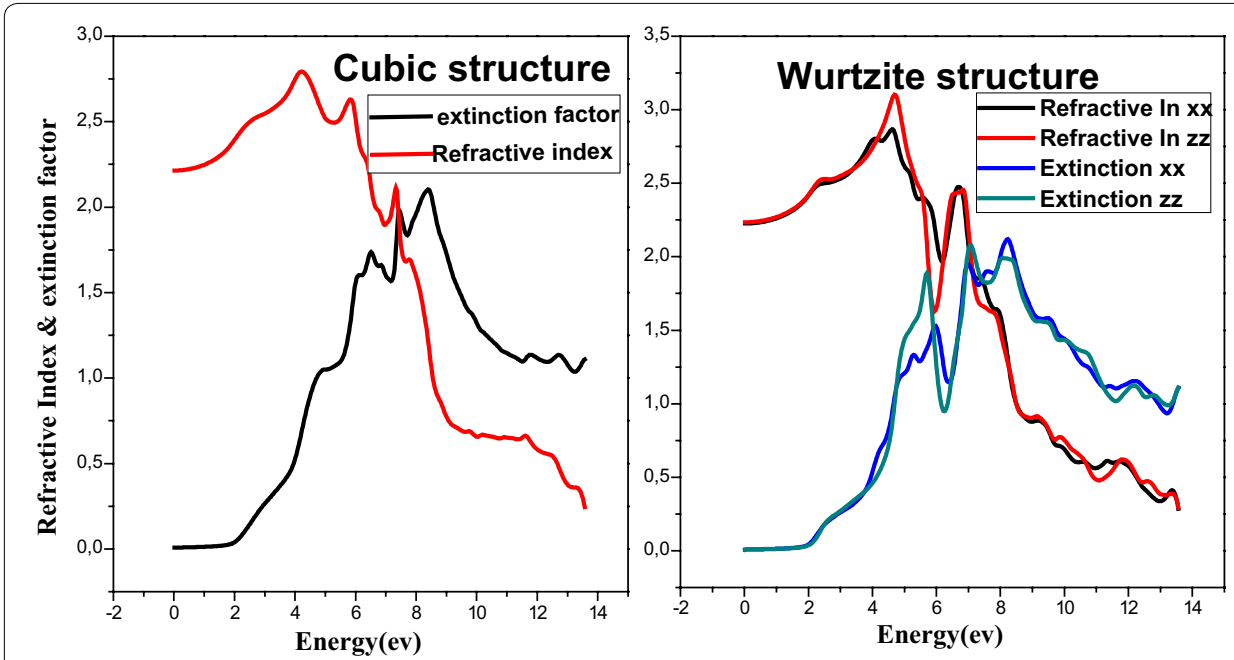

Fig. 6 Refractive index and extinction coefficient of CdSe in different phases

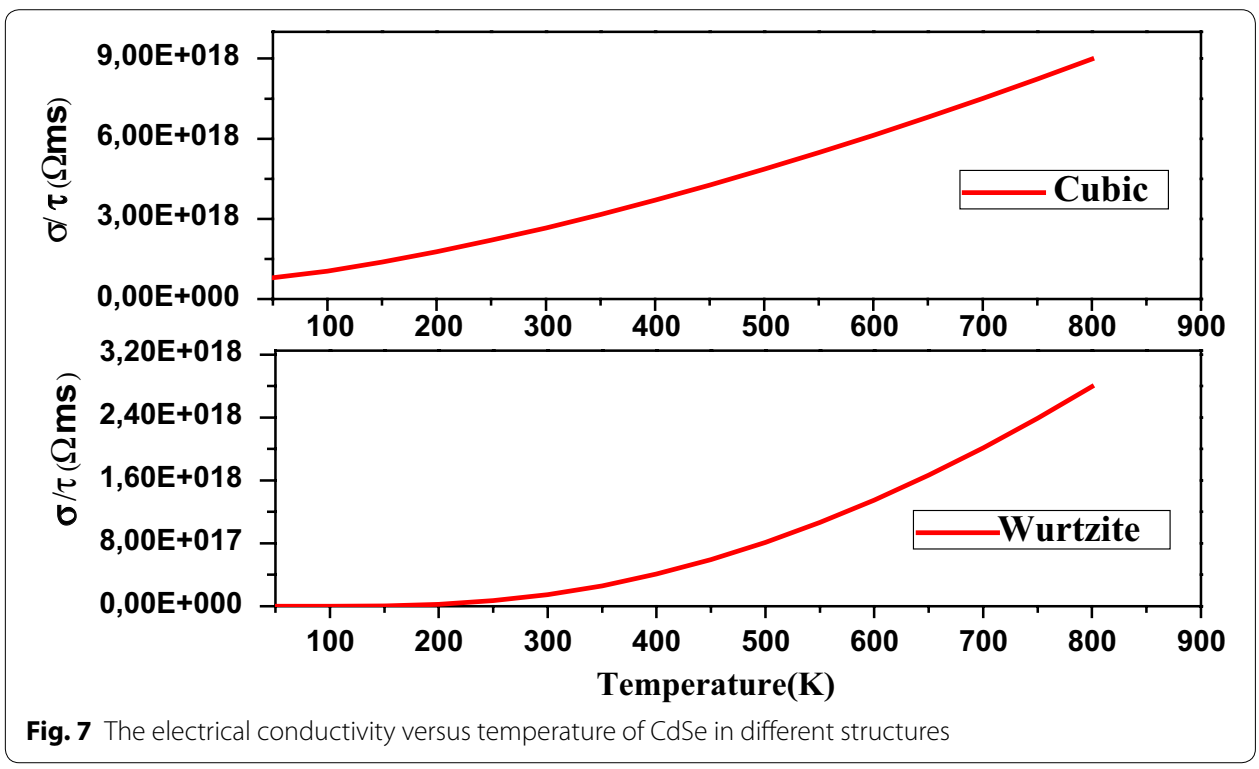

\section{Conclusion}

We have calculated the electronic, optical and electrical properties of CdSe in different phases, cubic and wurtzite model with different approximations. Using Boltztrap equations and FP-LAPW method with the generalized gradient approximation (GGA), local density approximation and $\mathrm{mBJ}$ approach. The calculations show that the cubic structure is isotope while for wurtzite structure the propagation of radiation $h v$ is different in $\mathrm{xx}$ and $\mathrm{zz}$ directions.

The decreases of absorption coefficient in the visible light, a stable transmittance which reach $80 \%$ in the visible light range beyond $400 \mathrm{~nm}$ were showed. We have investigated also that with increasing temperature, the electrical conductivity increases. The optical absorption is significant in the ultraviolet field. We have shown favorable results 
between calculations and the existing experimental data, these results confirm the quality that can present CdSe to be used as suitable material in optoelectronic and photovoltaic applications.

Authors' contributions

All authors have contributed equally to this work. All authors work in our laboratory and they have contributed to this work. All authors read and approved the final manuscript.

\section{Acknowledgements}

We would like to thank Mr. P. Blaha and Mr. K. Schwarz for the WIEN2k code that we used for this study. We would like also to thank Katarzyna Kula for the correction that she made to our manuscript.

\section{Compliance with ethical guidelines}

\section{Competing interests}

The authors declare that they have no competing interests.

Received: 6 May 2015 Accepted: 7 September 2015

Published online: 24 September 2015

\section{References}

Abbassi A, Ez-Zahraouy H, Benyoussef A (2015) First principles study on the electronic and optical properties of Al and Si- doped ZnO with GGA and mBJ approximations. Opt Quant Electron 47:1869-1880

Akinci Ö, Gürel HH, Ünlü H (2009) Semi-empirical tight binding modelling of CdSTe/CdTe, ZnSSe/ZnSe and ZnSSe/CdSe heterostructures. Thin Solid Films 517:2431-2437

Beaulac R, Paul Archer I, Stefan Ochsenbein T, Daniel Gamelin R (2008) Mn²+ doped CdSe quantum dots: new inorganic materials for spin-electronics and spin-photonics. Adv Funct Mater 18:3873-3891

Blaha P, Schwarz K (2006) WIEN2k. Vienna University of Technology, Austria

Blaha P, Schwarz K, Madsen GKH, Kvasnicka D, Luitz J (2011) WIEN2k an augmented plane wave + local orbitals program for calculating crystal properties; Karlheinz Schwarz, Techn. Universität Wien, Austria

Fuentes-Hernandez C, Suh DJ, Bernard Kippelen, Marder Seth R (2004) High-performance photorefractive polymers sensitized by cadmium selenide nanoparticles. Appl Phys Lett 85:534-536

Kale RB, Lokhande CD (2005) Band gap shift, structural characterization and phase transformation of CdSe thin films from nanocrystalline cubic to nanorod hexagonal on air annealing. Semicond Sci Technol 20:1-9

Karl W, Frese Jr (1982) A high efficiency singlecrystal CdSe photoelectrochemical solar cell and an associated loss mechanism. Appl Phys Lett 40:275-277

Klude M, Passow T, Heinke H, Hommel D (2002) Electro-optical characterization of CdSe quantum dot laser diodes. Phys Status Solidi B 229:1029-1032

Madsen GKH, Singh DJ (2006) BoltzTraP. A code for calculating band-structure dependent quantities. Comput Phys Commun 175:67-71

Marcel Bruchez Jr, Moronne M, Gin P, Weiss S, Paul Alivisatos A (1998) Semiconductor nanocrystals as fluorescent biological level. Science 281:2013-2016

Matsumura N, Endo H, Saraie J (2002) Fabrication of ZnSe diodes with CdSe quantum-dot layers by molecular beam epitaxy. Phys Status Solidi B 229:1039-1042

Merad AE, Kanoun MB, Merad G, Cibert J, Aourag H (2005) Full-potential investigation of the electronic and optical properties of stressed CdTe and ZnTe. Mater Chem Phys 92:333-339

Merad AE, Kanoun MB, Goumri-Said S (2006) Ab initio study of electronic structures and magnetism in ZnMnTe and CdMnTe diluted magnetic semiconductors. J Magn Magn Mater 302:536-542

Murray CB, Norris DJ, Bawendi MG (1993) Synthesis and characterization of nearly monodisperse CdE (E=S, Se, Te) semiconductor nanocrystallites. J Am Chem Soc 115:8706-87015

Ninomiya S, Adachi S (1995) Optical properties of wurtzite CdS. J Appl Phys 78:1183-1190

Perdew JP, Burke K, Ernzerhof M (1996) Generalized gradient approximation made simple. Phys Rev Lett 77:3865-3868

Slater JC (1951) Simplification of the Hartree Fock method. Phys Rev 81:385-390

Suresh S, Arunseshan C (2014) Dielectric properties of cadmium selenide (CdSe) nanoparticles synthesized by solvothermal method. Appl Nanosci 4:179-184

Uthana S, Reddy PJ (1981) Electrical properties of CdSe thin films. J Phys Status Solidi A 65:K113-K115

Ziman JM (2001) Electrons and phonons. Oxford University Press, New York 